\title{
LA COLECCIÓN DE ROLLOS DE PIANOLA DE LA FAMILIA FERNÁNDEZ-SHAW: UN ESTUDIO PRELIMINAR
}

\section{THE PIANO ROLLS COLLECTION OF THE FERNÁNDEZ-SHAW FAMILY: A PRELIMINARY STUDY}

\author{
Esther Burgos Bordonau \\ Departamento de Biblioteconomía y Documentación, \\ Universidad Complutense de Madrid \\ eburgos@ucm.es \\ ORCID iD: https://orcid.org/0000-0003-3831-9693 \\ Antonio Carpallo Bautista \\ Departamento de Biblioteconomía y Documentación, \\ Universidad Complutense de Madrid \\ acarpall@ucm.es \\ ORCID iD: https://orcid.org/0000-0001-7382-0649
}

\begin{abstract}
Resumen
El objetivo de este trabajo es el de acercar al lector a una singular colección de materiales musicales, el fondo de rollos de pianola de la familia Fernández-Shaw. Los herederos de tan insignes libretistas (Carlos, Guillermo y Rafael) han mantenido durante más de un siglo una interesante y modesta colección, formada por 223 rollos de pianola, que constituyen una curiosa selección de obras del género zarzuelístico en la que los libretistas participaron. A través de este estudio ofrecemos una visión general del contenido de los rollos, los géneros más comunes presentes en la colección y otras singularidades, propias de los documentos y dignas de mención.
\end{abstract}

\section{Palabras clave}

Carlos Fernández-Shaw / Guillermo Fernández-Shaw / Rafael Fernández-Shaw / rollo de pianola / Zarzuela / Oriental / Victoria / Aeolian Company

\section{INTRODUCCIÓN}

El presente trabajo, que como reza el propio título es un estudio preliminar, es el resultado del contacto establecido con los herederos de la familia Fernández-Shaw, cuyos ascendientes fueron indudables figuras de primer orden del teatro lírico español desde mediados del siglo XIX hasta un siglo más tarde. Fruto del encuentro académico e investigador del último Congreso de Musicología de la SEdeM, celebrado en Madrid en 2016, algunos profesores de la Facultad de Ciencias de la Docu-

\begin{abstract}
The aim of this work is to approach the reader closer to a singular collection of musical materials, the background of piano rolls of the Fernández-Shaw family. The heirs of such distinguished librettists (Carlos, Guillermo and Rafael) have kept for more than a century an interesting and modest collection, consisting of 223 piano rolls, that constitute a curious selection of works of Zarzuela in which the authors participated. Through this study we offer an overview of the content of the rolls, the most common genres present in the collection and also other singularities, proper of the documents worth of mention.
\end{abstract}

\section{Keywords}

Carlos Fernández-Shaw / Guillermo Fernández-Shaw / Rafael Fernández-Shaw / piano roll / Zarzuela / Oriental / Victoria / Aeolian Company

mentación de la Universidad Complutense de Madrid tuvimos la oportunidad de contactar con otros investigadores que nos abrieron el camino hacia esta ilustre familia y a su inesperada, desconocida e interesante colección de rollos de pianola.

Precisamente, el trabajo que presentamos en aquel evento musicológico versaba sobre una parte de la colección de rollos de pianola de la Biblioteca Nacional de España. De hecho, llevamos ya varios años trabajando este campo que aún permanece inédito y virgen para su estudio e investigación. Por esta razón fue de especial interés contactar con personas, investigadores de prestigio, que no sólo tenían 
amistad con las descendientes y herederas de los Fernández-Shaw sino que nos abrieron literalmente el camino a su casa y colección.

A partir del primer contacto con una de las herederas de los Fernández-Shaw, el camino para conocernos y descubrir su colección (y su pianola, heredada del abuelo Guillermo) fue muy fácil y rápido. De hecho, tras una primera toma de contacto y una visita al domicilio de esta persona, pronto vimos el material allí acumulado -sin demasiado orden ni concierto- y decidimos proponer un convenio de colaboración para su inventariado, estudio, clasificación y posterior catalogación. Rápidamente la propietaria de los rollos aceptó el reto y accedió a cedernos la colección en depósito durante un semestre. Empezaron los trámites y muy pronto la tarea.

A finales de enero de 2017 ya tuvimos en la sede de nuestra facultad los 223 rollos que componen la colección FernándezShaw. El grupo de investigación BIBLIOPEGIA (reconocido por sus trabajos sobre el libro antiguo y las encuadernaciones artísticas) iba a acometer la importante tarea de identificar todos y cada uno de estos singulares materiales, realizar un estudio de la colección en su conjunto y también en el momento de su edición y, por último y no menos importante, una catalogación precisa de todos y cada uno de los ejemplares. Para este último apartado nos hemos guiado y seguido el ejemplo utilizado por la Biblioteca Nacional de España aunque conservando nuestras propias características a la hora de plantear los campos de la ficha catalográfica.

El traslado de los materiales consistió en el envío de diez cajas con los rollos "sin ordenar". La primera de las tareas fue la realización de un primer inventario. Debíamos conocer qué teníamos y lo básico de los autores y las materias. Casi todos los rollos, salvo algunas excepciones, estaban en muy buen estado. Esto permitía su fácil reconocimiento en cuanto al estado de las cajas, las etiquetas a modo de tejuelo en las propias cajas así como las mismas en el interior o "portada" del rollo. Incluso ese buen estado es el que actualmente permite y facilita su reproducción en la pianola y su escucha. Sin embargo, otros aparecieron algo dañados, carentes de suficiente información y/o de anillas u otros elementos que permitiesen el perfecto agarre del ejemplar al mecanismo de la pianola y, por tanto, quedaba imposibilitada su reproducción. Fue obligado poner en conocimiento de la propietaria de la colección, a través del estado de los rollos que fuimos describiendo, cuáles de éstos necesitaban restauración para poder ser reproducidos. De toda la colección, tan sólo un rollo fue descartado para su descripción, al carecer éste de cualquier tipo de información literaria en el mismo. Ni la caja, ni la propia portada del rollo, presentaban información alguna para su identificación.

\section{METODOLOGÍA}

Recibimos en la sede de la Facultad de Ciencias de la Documentación diez cajas con los materiales sonoros, sin ningún orden preestablecido, y se acordó ir colocando los rollos según iban saliendo de las cajas. Se habilitó un pequeño despacho para realizar esta tarea y se habilitó un armario ex profeso para poder guardar bajo llave los materiales.

Se fue colocando una etiqueta identificativa de la caja de donde salían los rollos y, a cada uno de éstos, la consiguiente etiqueta con un número currens $(\mathrm{C} 1 / 1)$ para su posterior identificación en el inventario. Cuando tuvimos todos colocados y dispuestos a la vista, comenzó la tarea de identificación e inventariado. El inventario consistió en anotar autor, título y editor, como puede verse en la imagen.

\begin{tabular}{|c|c|c|}
\hline AUTOR & TÍTULO & EDITOR \\
\hline \multicolumn{3}{|l|}{ CAJA 1} \\
\hline VIVES, Amadeo & Doña Francisquita & VICTORIA \\
\hline SERRANO, José & $\begin{array}{l}\text { ¿Valencia canta? A la virgen de los } \\
\text { desamparados }\end{array}$ & ORIENTAL \\
\hline VIVES, Amadeo & El Duquesito. Coro de Richeliu & ORIENTAL \\
\hline SERRANO, José & El príncipe carnaval. Fox-trot de los martillos & ORIENTAL \\
\hline MORENO TORROBA & La marchenera. Las modas & DIANA \\
\hline GUERRERO, Jacinto & D. Quintín el amargao & ORIENTAL \\
\hline ALONSO, F & Las niñas de mis ojos. Fox del "Clemente" & ORIENTAL \\
\hline VIVES, Amadeo & Bohemios. Escena y Coro & ORIENTAL \\
\hline JUARRANZ & La Giralda. Paso-doble andaluz & ORIENTAL \\
\hline SERRANO y VALVERDE & El príncipe carnaval. (Escena del opio) Fox trot & ORIENTAL \\
\hline GUERRERO, Jacinto & $\begin{array}{l}\text { "Lo que va de ayer a hoy". Schottish de los } \\
\text { guardias }\end{array}$ & ORIENTAL \\
\hline GUERRERO, Jacinto & Las Alondras. Himno de los estudiantes & DIANA \\
\hline $\begin{array}{l}\text { PADILLA } \\
\text { Pation }\end{array}$ & La bien amada. Marcha & ORIENTAL \\
\hline ALBENIZ & Iberia. N. 2. Almería & THE AEOLIAN \\
\hline USANDIGAZA, José María & Las golondrinas. Pantomina & THE AEOLIAN \\
\hline MORENO TORROBA, Federico & La Mari-Blanca. Fado & ORIENTAL \\
\hline MILLÁN, Rafael & La Severa. Fado & VICTORIA \\
\hline MILLÁN, Rafael & La Severa. Romanza del Tenor & VICTORIA \\
\hline GRANADOS, Enrique & Escenas románticas & VICTORIA \\
\hline
\end{tabular}

Fig. 1. Primera página del inventario inicial

Realizada esta primera tarea, empezamos con el diseño y la adaptación de la ficha catalográfica a aquellos campos que considerábamos importantes y necesarios para una correcta descripción del material siguiendo para ello la norma ISBD consolidada [Descripción bibliográfica (2010)] y las Reglas de catalogación españolas [Reglas de Catalogación (2010)]. En consecuencia, los campos seleccionados fueron los siguientes:

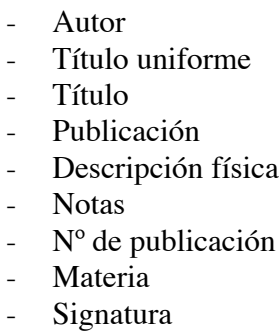

El referente fue la ficha bibliográfica de la Biblioteca Nacional de España aunque con nuestras adaptaciones y campos necesarios. Básicamente cabría decir que usamos las principales áreas de descripción aunque en el área de notas, por ejemplo, decidimos incluir información que consideramos relevante. Muchos de los rollos presentaban indicaciones de ejecución de dinámicas (Metrostyle ${ }^{1}$ o Themodist ${ }^{2}$ ), otros también llevaban escrito el tempo de la obra, incluso

1 Véase: Catalogue de musique pour pianola et pianola-piano music du métrostyle. Tomo I....

2 Véase: Catalogue de Musique pour Pianola et Pianola=Piano a 88 notes. Tome II... 
algunos los autores de los textos (en caso de tratarse de adaptaciones de obras líricas o canciones) y el texto, es decir la letra de la canción cuando -excepcionalmente- ha aparecido para nuestra sorpresa.

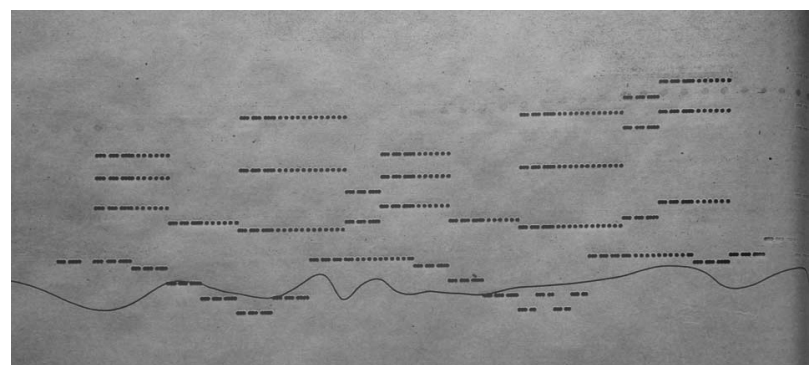

Fig. 2. Línea roja de Metrostyle Sig. 189/USA Las golondrinas (Fotografía de Juan Miguel Sánchez Vigil)

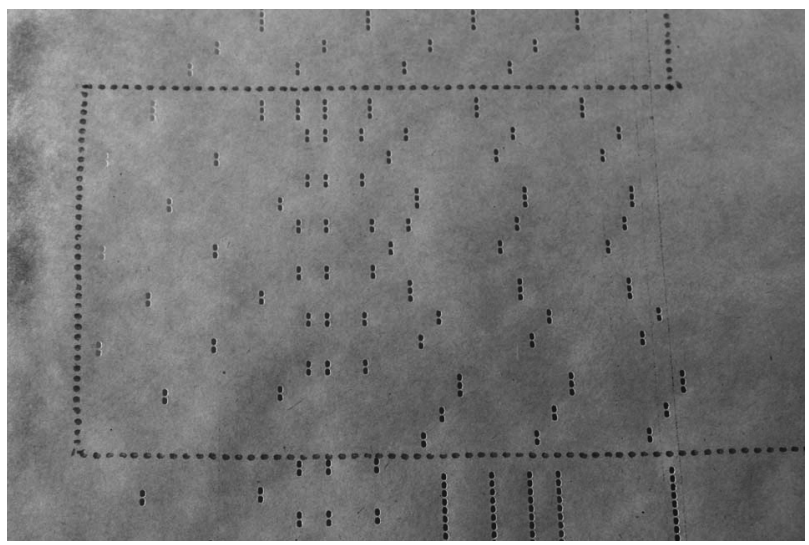

Fig. 3. Ejemplo de Themodist Sig. 103/GUE Las alondras (Fotografía de Juan Miguel Sánchez Vigil)

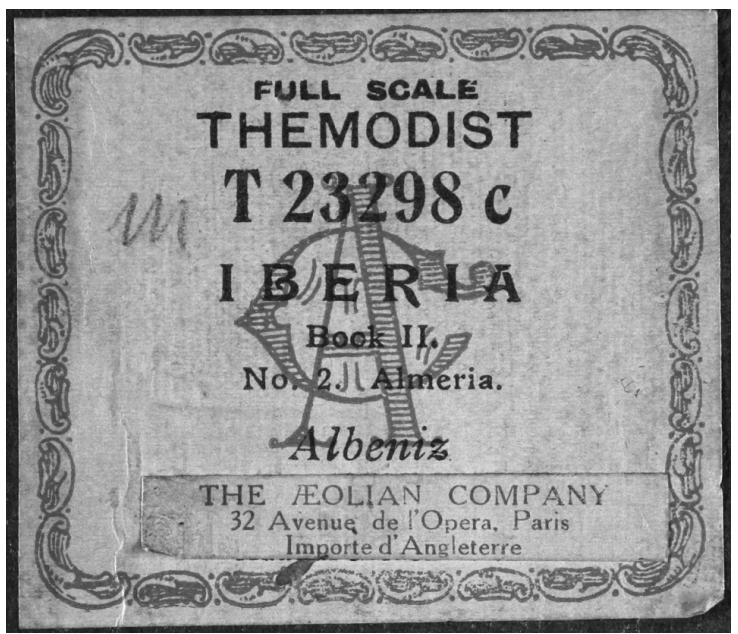

Fig. 4. Modo de reproducción indicado en la portada Sig. 3/ALB Iberia

(Fotografía de Antonio Carpallo Bautista)
En relación al Autor, el punto de acceso es el apellido/s y nombre del compositor, seguido y entre paréntesis de los años de nacimiento y muerte, como por ejemplo: Beethoven, Ludwig van (1770-1827).

Para el Título uniforme ${ }^{3}$, se siguieron estrictamente las Reglas de catalogación anteriormente citadas. A modo de ejemplo podemos citar la obra de Ludwig van Beethoven, cuyo título propiamente dicho es Sinfonía $n^{o} 5$ en Do menor, correspondiéndole como Título uniforme: [Sinfonías, $n^{\circ}$ 5, op. 67, do menor. Allegro (3er. Movimiento); arr.].

En cuanto al campo de Título además de este, se añade, siempre siguiendo la normativa, la mención de responsabilidad de la obra tal y como aparece en la fuente, compuesta en este caso por el nombre de compositor o compositores si hay más de uno. Utilizando el ejemplo anterior en este campo la obra quedaría reflejada de la siguiente manera: Sinfonía $n^{\circ} 5$ en Do menor / Beethoven .

En el campo de Publicación se refleja el lugar, el editor y el año de publicación. En este último elemento normalmente no siempre se anota un año concreto ya que este no suele aparecer en el documento, y debido a la dificultad en muchas ocasiones de la consulta de los catálogos editoriales, se ha datado, bien a partir de la fecha de composición o estreno de la obra y/o el periodo de producción de la editorial. Para indicar un año aproximado se ha utilizado la abreviatura de circa (alrededor de) como [ca. 1905], y para periodos más amplios la fórmula que se indica en el siguiente ejemplo: Madrid: Oriental, [entre 1905 y 1936].

En la Descripción física se indica el número de rollos, el número de notas para el que está compuesto $(65 \ldots . .88)$, que se corresponde con la extensión del teclado. En la colección que estamos tratando los rollos suelen ser de 88 notas, ya que esta cifra se normaliza a partir de 1908 dejando claro que las composiciones debían ser realizadas para esta extensión del teclado. Y ya como último elemento se indican las dimensiones del soporte. Un ejemplo de descripción del campo sería el siguiente: 1 rollo de pianola : 88 notas $; 32 \mathrm{~cm}$.

Respecto al campo de Notas, este ha sido el más variado aunque en primer lugar se ha indicado el tempo o velocidad del rollo, seguido, si procedía, del sistema Full Scale Themodist, que es el que permitía separar la melodía del acompañamiento ofreciendo un volumen diferente para cada voz. Otra nota, a nuestro juicio interesante, fue la observación de instrucciones de uso del soporte en diferentes idiomas como ha sucedido con los rollos de la Casa Editorial Victoria. A continuación, y aunque no es habitual en la descripción de los catálogos de rollos, hemos optado por incluir a los autores de los libretos de las obras, fundamentalmente de las zarzuelas. Procedimos así por tratarse

3 En líneas generales es aquél que permite reunir en un catálogo obras aparecidas bajo distintos títulos o lenguas, garantizando así su identificación. Véase: González Valle, José Vicente: Ezquerro Esteban, Antonio; Iglesias Martínez, Nieves; Gosálvez Lara, Carlos José; y Crespí González, Joana: Répertoire International des Sources Musicales (RISM). Normas internacionales para la catalogación de fuentes musicales históricas. (Serie A/II, Manuscritos musicales, 1600-1850), 1996, Madrid, Arco/Libros. 
de una colección privada en la que más de un tercio de la misma tiene por autores literarios a los Fernández-Shaw, cuyos herederos son los propietarios de la colección ${ }^{4}$. Entendimos que estos autores debían encontrarse destacados en nuestro catálogo. Al haber seguido este criterio para la saga de los Fernández-Shaw, consideramos siempre que nos fue posible, hacerlo con el resto de obras y autores literarios. Finalmente se indicaron, en la mayoría de los casos, las fechas de composición y/o estreno de la obra así como el lugar del mismo. Valga como ejemplo la siguiente descripción: En el rollo: "80 allegro". - Instrucciones para el uso del rollo en la pianola en francés, inglés y español. Libreto de Federico Romero y Guillermo Fernández Shaw. Estrenada el 15 de noviembre de 1928 en el Teatro Apolo de Madrid. Impreso en caja catálogo de selecciones de zarzuelas.

Este último ejemplo es uno de los muchos que hemos hallado en la colección que tienen la particularidad de tener instrucciones, repertorio u otro tipo de indicaciones. Concretamente se trata de la obra de Amadeo Vives Los Flamencos, una selección. (Fig. 5).

Otra curiosidad la hemos hallado en la obra Escenas Románticas de Enrique Granados. Esta conserva en su estuche un albarán de la compra donde se indica que el precio fue de 11 pesetas y también la fecha de adquisición (Fig. 6).

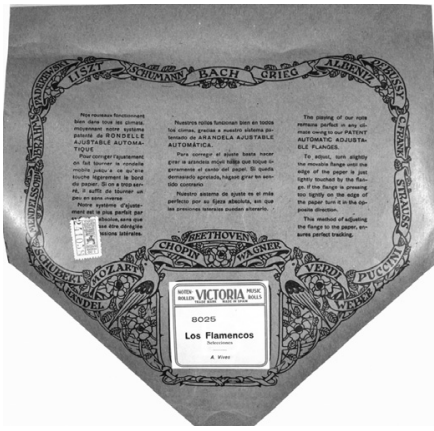

Fig. 5. Imagen del rollo

Victoria con instrucciones de uso en 3 idiomas

219/VIV Los flamencos

(Fotografía de Antonio Carpallo Bautista)

Otra de las curiosidades más notables que encontramos en esta colección fue un rollo con indicación de "cantor". Este tipo de rollos, más conocidos como text-rolls, fueron una novedad muy curiosa de la época pues permitía al que ejecutaba el rollo cantar simultáneamente una canción que aparecía impresa en el mismo. La técnica, muy precisa en la elaboración de las

4 Probablemente esta fuera una razón de peso que les llevara a recopilar y coleccionar, en formato rollo de pianola, sus propias obras del teatro lírico puesto que entre los tres autores, Carlos, Guillermo y Rafael cubrieron casi un siglo de historia de la zarzuela con sus textos. perforaciones, permitió igualmente imprimir en el lugar exacto la sílaba o palabra a cantar junto con la música que iba sonando al mismo tiempo. El resultado fue un anticipo del actual karaoke como bien han señalado otros autores anteriormente ${ }^{5}$. El rollo lo hemos identificado en nuestro catálogo con la signatura 160/ PAD (Ça--c'est) y puede verse en las siguientes figuras 7 y 8 .

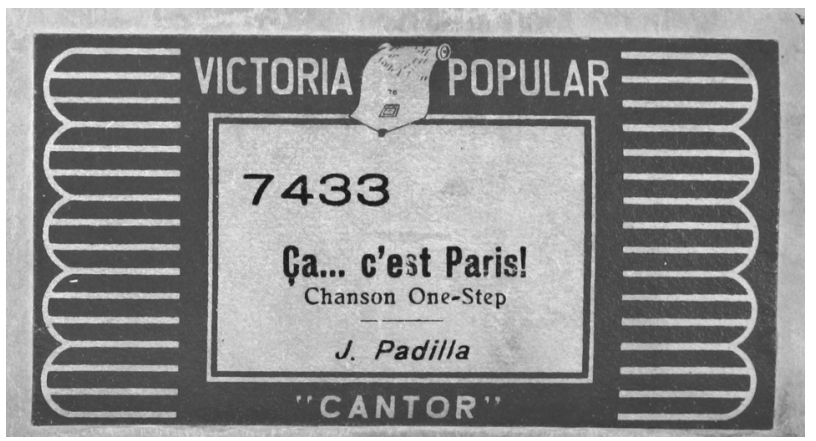

Fig. 7. Etiqueta de Rollos Victoria Popular con indicación de texto cantable "CANTOR" (Fotografía de Antonio Carpallo Bautista)

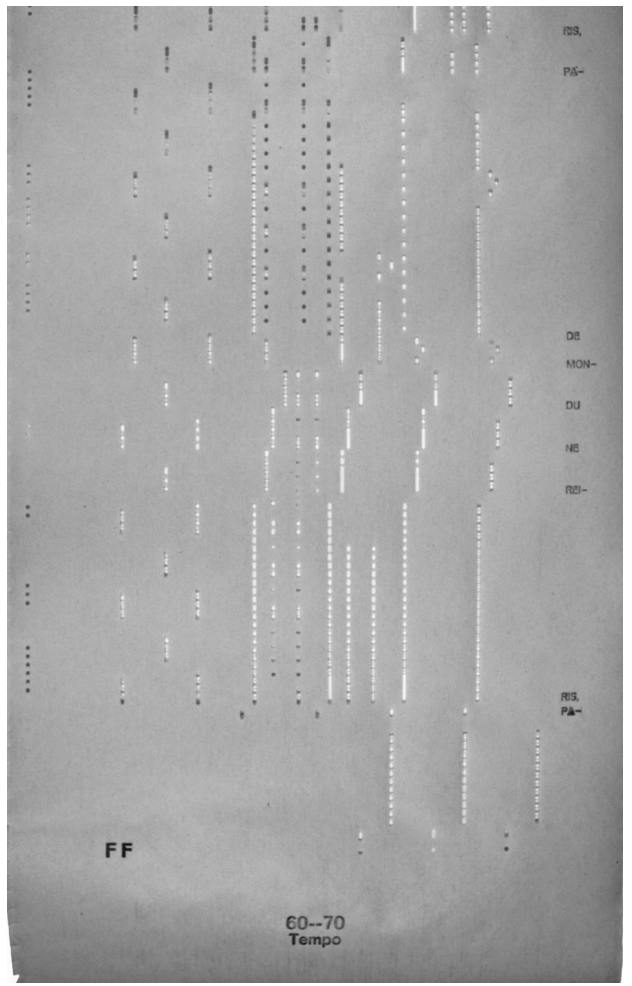

Fig. 8. Imagen del rollo con el texto cantable impreso (Fotografía de Antonio Carpallo Bautista)

5 ROQUER (2014): 142. 
El Número de publicación se corresponde con el número de identificación asignado por la casa editorial. Como ejemplo: E. 1063 The Aeolian Company.

El siguiente campo corresponde a la Materia de la obra, donde sobresale el género de la zarzuela, aunque también se dan otros como por ejemplo chotis, fox-trot, cuplé, tango, charlestón, mazurca, suite, sinfonía, etc., seguidos todos ellos del término aclaratorio arreglos para pianola. No debe olvidarse que a pesar de la gran colección de zarzuelas arregladas para ser interpretadas mediante este soporte, muchos otros estilos musicales se adaptaron a la perfección a este sistema.

Signatura es el último de los campos descrito. En esta ocasión y puesto que la colección estaba carente de cualquier tipo de numeración, ordenamiento o clasificación, optamos por identificar las obras procediendo de esta manera: adjudicación de un número currens seguido de las tres primeras letras del apellido del compositor en mayúsculas y, entre paréntesis, la parte más identificativa del título de la obra, conservando los artículos como elemento inicial. A modo de ejemplo podemos mencionar la obra de Enrique Granados, anteriormente citada Escenas románticas que fue identificada de la siguiente manera: 74/GRA (Escenas). Esta ordenación permite facilitar la colocación definitiva de los rollos y su rápida consulta en el domicilio de la familia propietaria. Cuando optamos por esta clasificación, que sin duda nos pareció la más adecuada, pensamos en todo momento que se trataba de una colección cerrada y que no habría más rollos que añadir a la misma, por esta misma razón los propietarios aceptaron de buen grado nuestra decisión.

File Maker Pro, versión 15, fue la aplicación informática escogida para poder llevar adelante nuestro catálogo. Es un programa que ofrece una gran versatilidad en el tratamiento de los datos y su manejo es relativamente sencillo para el usuario medio. A continuación mostramos un ejemplo de ficha catalográfica elaborada con dicho programa:

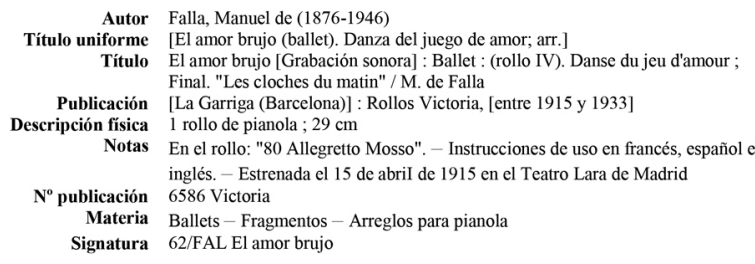

Fig. 9. Ejemplo de ficha catalográfica en File Maker Pro

\section{LA COLECCIÓN Y SUS CARACTERÍSTICAS}

Como ya señalábamos en el resumen inicial, esta modesta pero a la vez interesante colección se compone de 223 rollos de los que 50 se corresponden con obras de la autoría literaria de Guillermo Fernández-Shaw, entre los que destacan: Doña Francisquita (con 11 ejemplares), El caserío, El dictador, La canción del olvido, La meiga, La rosa del azafrán, La severa, La villana, Las alondras, Los flamencos y Luisa Fernanda. Tres títulos co- rresponden a Carlos Fernández-Shaw y éstos están repartidos en 5 ejemplares: Don Lucas del Cigarral, La chavala y La revoltosa. Podría decirse que el grueso de la colección está formado por selecciones y/o arreglos de Zarzuela, 92 ejemplares corresponden a esta materia. Y ¿qué hay del resto?

No debe olvidarse que los años "dorados" de la pianola fueron las tres primeras décadas del siglo XX y durante éstas, muchos géneros bailables inundaron los salones de recreo, los cafés y otros lugares de esparcimiento público. La pianola solía ser un instrumento adecuado para estos espacios públicos. Por supuesto, las pianolas ocuparon también salones de la alta burguesía y, por esta razón, hallamos abundante reportorio de música bailable y otros géneros de moda en aquellos tiempos. Los "felices años 20" también quedan reflejados en el repertorio disponible para este tipo de soporte. Se han contabilizado hasta 34 géneros diferentes dentro de la colección Fernández-Shaw. Por mencionar sólo algunos de los más representativos y dejando a un lado la Zarzuela (el principal ya mencionado) citaremos los siguientes por orden de representatividad: Fox-Trots, Sainetes, Suites, Sinfonías, Óperas, Música para piano, Operetas y Bailables en general (one-steps, charlestones, valses, pasodobles, cuplés, tangos). Incluso hay también himnos militares y rapsodias.

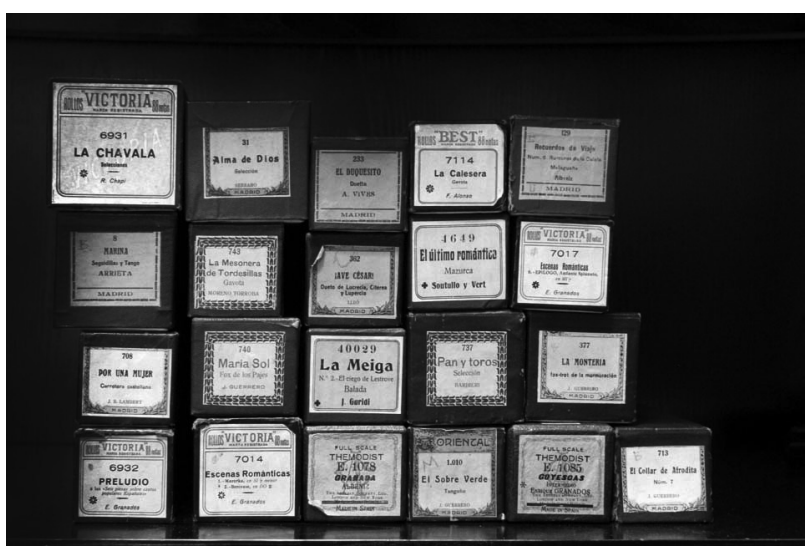

Fig. 10. Imagen de varias cajas de rollos con sus etiquetas (Fotografía de Juan Miguel Sánchez Vigil)

Otro aspecto también relevante y que ya apuntamos en nuestra comunicación al último Congreso Nacional de Musicología (Congreso de la SEdeM, Madrid 2016) es el tema de las editoriales de rollo de pianola. Sigue pendiente la realización de un estudio en profundidad sobre quiénes y cuáles fueron las casas productoras de estos soportes tan singulares. El estudio de esta colección también nos ha servido para ver cuáles fueron las más representadas y con qué tipo de repertorio trabajaron ${ }^{6}$.

6 Al hilo de este comentario hemos consultado y trabajado con algunos de los catálogos actualmente disponibles en la Biblioteca Digital Hispánica que hacen referencia a rollos de la colección aquí estudiada y que nos permiten conocer más en detalle el repertorio que ofrecían 
Precisamente y debido a nuestro conocimiento previo de las principales marcas españolas, pudimos comprobar que éstas están representadas casi todas, siendo la más numerosa la marca Oriental con 101 ejemplares dentro de la colección. Este dato llamó mucho nuestra atención pues sabemos, por estudios previos, que Rollos Musicales Victoria fue la pionera en la fabricación de rollos de pianola en España y probablemente también durante mucho tiempo, la primera suministradora en el mercado español e hispano-americano (Rollos Best). Las marcas nacionales presentes en la colección Fernández-Shaw son: Oriental (Fig. 13), Victoria, Diana (Fig. 14), Princesa (Fig. 12), Best, Victoria Popular (Fig. 16), Era y Mott (Fig. 15). Cabría añadir que Aeolian Company (Fig. 11), originaria de Estados Unidos, tuvo sucursales en Londres, París, Madrid y otras capitales. Los rollos que se fabricaron en España bajo este rótulo están también presentes en la colección y han sido considerados dentro de los de fabricación nacional. Junto a estos, la colección también tiene varios ejemplares extranjeros: 1 rollo de The Baldwin Company (EEUU) y varios rollos de The Aeolian Company producidos en Londres. Otro rollo interesante, también por ser el único, es el de la marca Armonic Rolls, de fabricación nacional. Creemos que estuvo hecho en Barcelona.

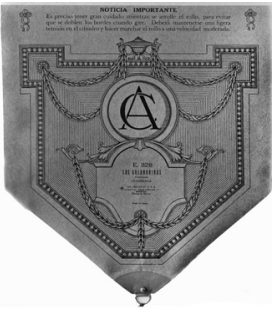

Fig. 11. Rollo Aeolian

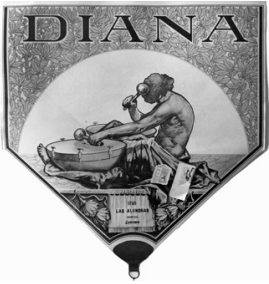

Fig. 14. Rollo Diana

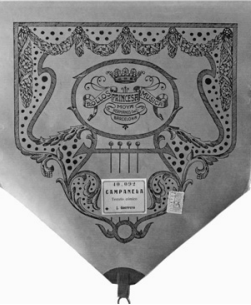

Fig. 12. Rollo Princesa

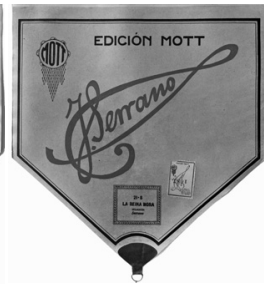

Fig. 15. Rollo Mott

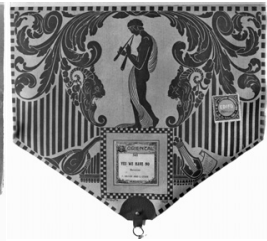

Fig. 13. Rollo Oriental

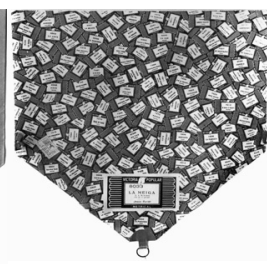

Fig. 16. Rollo Victoria Popular
A pesar de que muchos de los rollos de la colección parecían iguales, no todos lo eran. De hecho, las dimensiones de los soportes han sido también objeto de nuestra atención, como así hemos reflejado también en el área de Notas. Generalmente los

así como los precios de los ejemplares. En las referencias bibliográficas del final del texto pueden consultarse algunos de estos catálogos, casi todos pertenecientes a los primeros años del siglo XX. rollos son de $29 \mathrm{~cm}$. de largo aunque también hemos encontrado algunos de $32 \mathrm{~cm}$. Las indicaciones de las dinámicas, el tempo $(60,70,100$ o Allegro) e incluso la inserción de signos como calderones u otros términos impresos en el papel que se refieren al modo de hacer sonar el rollo, fueron también objeto de estudio y de consideración a la hora de consignar la información. Igualmente el número de notas para el cual estaba hecho el ejemplar, por lo general para 88 notas, que terminó convirtiéndose en el estándar aunque también en la colección hemos encontrado alguno para 65 notas, y las instrucciones de uso de los rollos explicadas, según las diferentes editoriales, en distintos idiomas, como hemos podido observar en las etiquetas de los rollos musicales Victoria ${ }^{7}$ o más claramente en los de España Musical ${ }^{8}$.
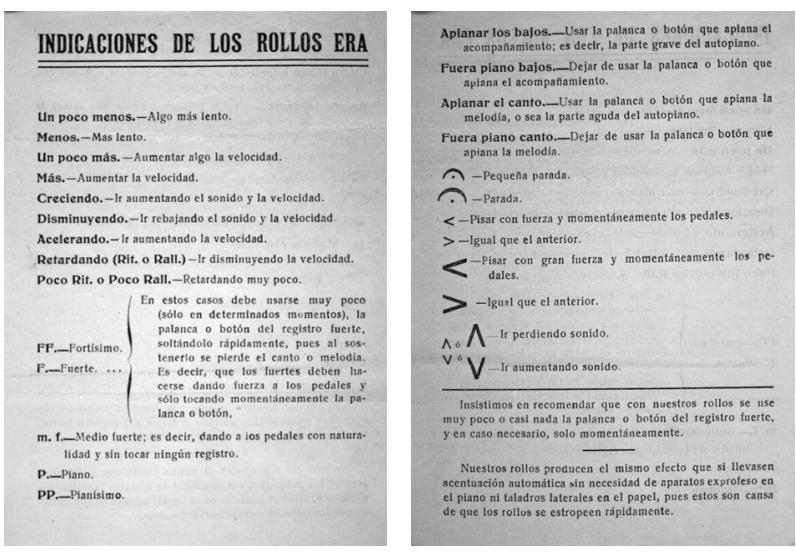

Fig. 17 y Fig. 18. Foto del folleto de Rollos Musicales ERA con instrucciones de uso (verso y reverso) (Fotografías de Antonio Carpallo Bautista)

\section{PRINCIPALES AUTORES Y OBRAS PRESENTES EN LA COLECCIÓN}

Jacinto Guerrero, Amadeo Vives y, a cierta distancia, José Serrano, son los autores principales presentes con sus obras en la colección. Guerrero destacó por ser un autor muy prolífico y la mayoría de sus obras fueron grandes éxitos de Zarzuela. Muchas de sus zarzuelas aparecen repetidas veces en la colección. Obras como La rosa del azafrán, Las alondras, La montería, El sobre verde, etc... están entre las más populares de la colección. Hay numerosos rollos con distintas partes de las obras, selecciones. También de él nos han llegado muchos otros sainetes y teatro lírico.

De Amadeo Vives nos encontramos con varios ejemplares de la zarzuela Doña Francisquita y también Bohemios, La villana, Los flamencos, Don Lucas del Cigarral (obra con texto de Luceño y Carlos Fernández Shaw, inspirada en la novela cer-

7 Véase Rollos Musicales Victoria, catálogo general,...(1929): 5

8 Véase Catálogo general de rollos de 88 y 65 notas y sus similares. España Musical, S.A....(1925): $9-13$. 
vantina de El Quijote). De José Serrano tenemos La canción del olvido, Alma de Dios, Moros y cristianos, Los de Aragón, La alegría del batallón... entre otras.

Jesús Guridi aparece en la colección con un número de obras similar a José Serrano. Con respecto a los autores clásicos, éstos también están presentes en la colección aunque en menor medida que los autores españoles, a excepción del caso de Ludwig van Beethoven quien presenta el mismo número que el compositor valenciano. Si bien es cierto que la mayoría de las composiciones pertenecen al género de la zarzuela, también hay obras clásicas como selecciones de sinfonías, conciertos y otras similares. Junto al autor alemán y dentro del repertorio tradicional encontramos también obras de Isaac Albéniz, Enrique Granados, Félix Mendelssohn, Frédéric Chopin, Rimski-Kórsakov y Manuel de Falla.

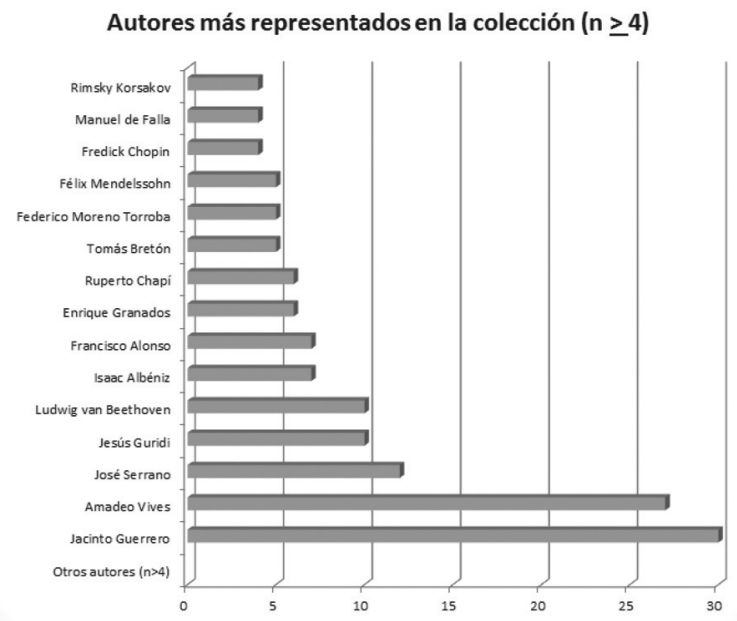

Fig. 19. Cuadro de los autores más representados de la colección

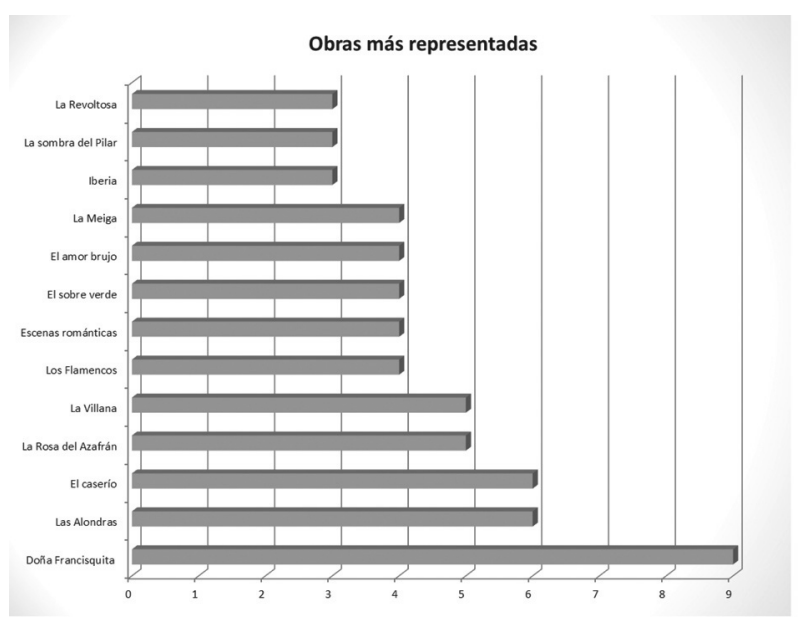

Fig. 20. Cuadro de las obras más representadas de la colección

\section{CONCLUSIONES}

La realización de esta tarea de ordenación, inventariado y catalogación de la colección de rollos de pianola de la familia Fernández-Shaw nos ha servido, entre otras cosas, para aprender mucho sobre este tipo de soportes a los que ya nos habíamos asomado en estudios previos realizados con la importante colección que posee la Biblioteca Nacional de España. Igualmente nos ha permitido ampliar nuestro conocimiento, no sólo en el modo de producir y hacer sonar estos soportes, sino también en conocer mejor cuál fue el detonante de que dichos rollos se empezaran a popularizar en nuestro país.

Como ya se señaló antes, el catálogo de la Biblioteca Nacional de España fue fundamental como herramienta de consulta para la elaboración de nuestro catálogo particular. Si bien no estaban todas las obras que teníamos entre manos, sí muchas de ellas y sus descripciones fueron clave para poder resolver aspectos descriptivos importantes. Incluso hemos podido añadir datos que no aparecen en las fichas de la BNE pero que sí hemos averiguado nosotros a lo largo de nuestra investigación (datos de autores literarios, fechas de composición y/o estreno de las obras), información que tuvimos que consultar a través de diferentes fuentes bibliográficas -obras de referencia, webs, libretos- que ahora permiten un mayor conocimiento de la obra que se tiene entre manos.

Las principales conclusiones a las que hemos llegado podrían resumirse de la siguiente manera. La pianola, instrumento que nace a finales del siglo XIX en EEUU y que pronto tuvo su correlato en España, vivió sus mejores tiempos durante los primeros cuarenta años del siglo XX, coincidiendo exactamente con la época de mayor esplendor de Guillermo Fernández-Shaw (1893-1965) y con la reunión de esta peculiar colección particular. Carlos Fernández-Shaw, el primero de la saga y padre de Guillermo y de Rafael, también tuvo que conocer el invento aunque falleció a comienzos del siglo XX (1911), cuando comenzaba la expansión y popularización del mismo. La pianola fue un instrumento que tuvo una importante difusión entre las clases medias y altas del país pues no estaba al alcance de cualquiera un aparato de estas características. Los primeros instrumentos conocidos fueron localizados en lugares públicos como cafés, salones, cines y otros centros de reunión.

Recordamos la conocida anécdota sobre el primer fabricante de rollos de pianola en España, Joan Baptista Blancafort. Este conoció a un tal Carlos Salvi -comerciante de la casa Angelus de Madrid- quien le vendió una pianola para su establecimiento, el balneario de aguas termales situado en la localidad barcelonesa de La Garriga. Con dicho instrumento la fama y el gusto por estar en el establecimiento crecería y de ahí surgió el posterior interés que el imparable Joan Baptista Blancafort tuvo por crear sus propios rollos, con repertorio más del gusto de los clientes que tenía la casa de baños ${ }^{9}$, entre otros a este ilustre señor que también después le ofrecería la representación de esta firma en toda Cataluña.

\footnotetext{
9 BLANCAFORT (1976): 86.
} 
De hecho, lo primero que se importó del extranjero y con lo que contamos es con repertorio clásico: Beethoven, Mendelssohn, Bach, Chopin, Meyerbeer, Wagner, etc. Al poco tiempo, tanto desde el propio balneario catalán como desde las primeras salas en donde se hizo sonar el instrumento, comenzó a demandarse un repertorio más acorde con el público del momento. Así fue como nació el gusto por la música popular en este formato y, sobre todo, la gran expansión y difusión del género lírico (zarzuelas, sainetes, operetas, operetas, revistas) a través del papel perforado. Con el tiempo se irían añadiendo otros estilos, más en la línea de la música de entretenimiento o música bailable.

Los catálogos de las casas editoriales han sido claves a la hora de conocer cuál era el repertorio editado y cómo era el gusto de la sociedad del momento. Hay numerosos catálogos, desde comienzos del siglo XX, de la casa Victoria, Diana, España Musical y tantas otras. Muchos están digitalizados y pueden consultarse a través de Biblioteca Digital Hispánica ${ }^{10} \mathrm{o}$ a través de la base de datos MATRIZ ${ }^{11}$.

Un último prejuicio que nos gustaría desterrar es aquel que presenta a la pianola como un instrumento totalmente mecánico y carente de intervención personal. Nada de esto era totalmente cierto. Hemos podido comprobar, a través del estudio de los propios rollos y de todas las indicaciones que se daban para su perfecta ejecución, que el pianolista sí que tenía algún protagonismo en la ejecución del rollo musical y que por tanto, a pesar de lo mecánico de la música, había bastantes matices que podía manipular y realizar a su gusto. Prueba de ello son todas las indicaciones que aparecen impresas en los propios rollos para que, si el sistema que tenía la pianola además lo permitía, el ejecutante pudiera tocar el rollo más o menos a voluntad, en lo que a dinámicas se refiere y, por supuesto, en lo relativo al tempo o velocidad.

El trabajo final relativo a esta colección, la publicación de un catálogo completo con las descripciones de los 223 rollos que componen el fondo, está en proyecto de elaboración. Dicha investigación irá acompañada del correspondiente estudio de la época y de la influencia de la saga de los Fernández-Shaw en el panorama musical y literario español.

Confiamos en que la publicación de este catálogo sirva para abrir brecha y animar a los musicólogos e investigadores a seguir avanzando en el conocimiento y descripción de nuestro rico patrimonio sonoro musical.

\section{BIBLIOGRAFÍA}

Best: rollos de música de 88 notas con acentuación y autopedal para toda clase de pianos combinados con aparatos pianistas: catálogo general, [ca. 1920], [Barcelona, Tip. Vda. de Luis Tasso]

Burgos Bordonau, Esther, 2016, "La colección de rollos de pianola de zarzuela de la Biblioteca Nacional de España: descripción y análisis preliminar", Actas del IX Congreso Nacional de la Sociedad Española de Musicología, Musicología en el siglo XXI: nuevos retos, nuevos enfoques, Madrid, SEDEM, [en prensa]

Burgos Bordonau, Esther; Carpallo Bautista, Antonio; Clausó García, Adelina, 2017, La colección de rollos de pianola de la familia Fernández-Shaw. Catálogo de la exposición. Madrid, Universidad Complutense de Madrid y Grupo de investigación Bibliopegia.

Burgos Bordonau, Esther; Carpallo Bautista, Antonio; Clausó García, Adelina, 2018, "Identificación, estudio y catalogación de la colección de rollos de pianola de la familia Fernández-Shaw”, Conocimientos sin fronteras: colaboración científica e institucional en Documentación e Información, Madrid, Facultad de Ciencias de la Documentación, Universidad Complutense de Madrid, 201-220.

Blancafort, Pere, 1976, La Garriga, el Balneari i jo. Barcelona, Ariel.

Descripción bibliográfica internacional normalizada: ISBD. Ed preliminar consolidada, 2010, Madrid, ANABAD.

Catalogue de musique pour pianola et pianola-piano music du métrostyle, Tomo I, 1905, París, The Aeolian Company, Annexe et Biblioteques.

Catalogue de Musique pour Pianola et Pianola=Piano a 88 notes. Tome II, 1914 , París, The Aeolian Company.

Catálogo general de rollos de 88 y 65 notas y sus similares. España Musical, S.A., fábrica de rollos de música para aparatos mecánicos y eléctricos de toda clase de perforaciones, [ca.1925], Cartagena, Imprenta y Enc. Viuda de Salvador Garnero.

González Valle, José Vicente: Ezquerro Esteban, Antonio; Iglesias Martínez, Nieves; Gosálvez Lara, Carlos José; y Crespí González, Joana, 1996, Répertoire International des Sources Musicales (RISM). Normas internacionales para la catalogación de fuentes musicales históricas. (Serie A/ II, Manuscritos musicales, 1600-1850). Madrid, Arco/ Libros.

Reglas de Catalogación, Ed. nuevamente rev. $7^{a}$ reimp. 2010 , Madrid, Ministerio de Cultura, Subdirección General de Publicaciones, Información y Documentación.

Rollos Musicales de 88 notas con acentuación y autopedal Princesa. Catálogo general, 1930. Barcelona, Moya y hermanos $\mathrm{S}$ en $\mathrm{C}$.

Rollos Musicales Victoria, catálogo general, 1929, La Garriga, Barcelona, Joan Baptista Blancafort.

Roquer González, Jordi, 2014, "El sons ocults del paper perforat", Revista Catalana de Musicologia, VII, 137-152.

Recibido: 26.09.2017

Aceptado: 16.04.2018

10 Véase. http://www.bne.es/es/Catalogos/BibliotecaDigitalHispanica/Inicio/index.html

11 Véase. http://www.aedom.org/matriz 